\title{
THE APPROXIMATION PROPERTIES OF MODIFIED $(p, q)$-GAMMA OPERATORS PRESERVING LINEAR FUNCTIONS
}

\author{
JING ZHANG ${ }^{1}$, WENTAO CHENG ${ }^{2, *}$, FENGLIN CHEN $^{2}$ \\ ${ }^{1}$ Department of Automation, Taiyuan Institute of Technology, Taiyuan China \\ ${ }^{2}$ School of Mathematics and Physics, Anqing Normal University, Anhui, China
}

\begin{abstract}
In this paper, we introduce a class of modified $(p, q)$-Gamma operators based on $(p, q)$-calculus that operators preserve not only constant functions but also linear functions. Then the moments of the operators are established and some local approximation theorems of these operators are discussed. Also, the rate of convergence and weighted approximation of these operators are studied by means of modulus of continuity. Furthermore, the Voronovskaya type asymptotic formula is investigated.
\end{abstract}

Keywords. $(p, q)$-integers; Modified $(p, q)$-Gamma operators, Rate of convergence; Weighted approximation.

\section{INTRODUCTION}

In 2007, Karsli [1] investigated and studied some approximation properties of the following linear positive operators (named Gamma operators)

$$
L_{n}(f ; x)=\frac{(2 n+3) ! x^{n+3}}{n !(n+2) !} \int_{0}^{\infty} \frac{t^{n}}{(x+t)^{2 n+4}} f(t) \mathrm{d} t, x>0 .
$$

In 2010, Karsli and Özarslan [2] established some approximation results for the above operators. The applications of the $q$-calculus in approximation theory recently attract much attention. In 2013, Cai and Zeng [3] defined the $q$-analogue of the Gamma operators (1.1) preserving $x^{2}$. In the same year, they also defined modified $q$-Gamma operators preserving linear functions [4]. In [5, 6], $q$-Gamma-Stancu operators and modified $q$-Gamma-Stancu operators were studied, respectively. Afterwards, with the generalization from the $q$-calculus to the $(p, q)$-calculus which has been used efficiently in many fields, such as, Algebras, CAGD and so on. Mursaleen, Khan and Khan first introduced the $(p, q)$-calculus in approximation theory and constructed the $(p, q)$-analogue of Bernstein operators [7] and $(p, q)$-Bernstein-Stancu operators [8].

\footnotetext{
${ }^{*}$ Corresponding author.

E-mail addresses: titzhjing1989@sina.com(J. Zhang), chengwentao_0517@163.com (W. Cheng), chenfenglin @ aqnu.edu.cn (F. Chen).

Received August 21, 2020; Accepted January 11, 2021.
} 
Recently, the $(p, q)$-analogue of some more operators have been studied in $[9,10]$. Up to the authors' knowledge, there is no result on the $(p, q)$-analogue of these operators defined in (1.1) preserving constant functions and linear functions (named modified $(p, q)$-Gamma operators).

In this paper, we will establish the local approximation theorems of the operators $\widehat{G}_{n}^{p, q}$, and give the estimates on the rate of convergence and the weighted approximation of theses operators.

Before introducing the operators, we mention certain definitions based on the $(p, q)$-calculus. We refer the reader to $[11,12,13]$. For any fixed real numbers $p>0$ and $q>0$, the $(p, q)$ integers $[n]_{p, q}$ are defined by

$$
[n]_{p, q}=p^{n-1}+p^{n-2} q+p^{n-3} q^{2}+\cdots+p q^{n-2}+q^{n-1}= \begin{cases}\frac{p^{n}-q^{n}}{p-q}, & p \neq q \neq 1 \\ n p^{n-1}, & p=q \neq 1 \\ {[n]_{q},} & p=1 \\ n, & p=q=1\end{cases}
$$

where $[n]_{q}$ denotes the $q$-integers and $n=0,1,2, \cdots$. The $(p, q)$-factorial $[n]_{p, q}$ ! are defined by

$$
[n]_{p, q} != \begin{cases}{[1]_{p, q}[2]_{p, q} \cdots[n]_{p, q},} & n \geq 1 \\ 1, & n=0 .\end{cases}
$$

The $(p, q)$-power basis is defined by

$$
(x \oplus y)_{p, q}^{n}=(x+y)(p x+q y)\left(p^{2} x+q^{2} y\right) \cdots\left(p^{n-1} x+q^{n-1} y\right) .
$$

Let $f$ be an arbitrary function. The improper $(p, q)$-integral of $f(x)$ on $[0, \infty)$ is defined as (see [14])

$$
\int_{0}^{\infty} f(x) \mathrm{d}_{p, q} x=(p-q) \sum_{n=-\infty}^{\infty} \frac{q^{n}}{p^{n+1}} f\left(\frac{q^{n}}{p^{n+1}}\right), 0<\frac{q}{p}<1 .
$$

Two different $(p, q)$-analogues of the exponential function named $e_{p, q}(x)$ and $E_{p, q}(x)$ (see [13]) are defined as follows:

$$
e_{p, q}(x)=\sum_{n=0}^{\infty} \frac{p^{\frac{n(n-1)}{2}} x^{n}}{[n]_{p, q} !}
$$

and

$$
E_{p, q}(x)=\sum_{n=0}^{\infty} \frac{q^{\frac{n(n-1)}{2}} x^{n}}{[n]_{p, q} !}
$$

which satisfy the equality $e_{p, q}(x) E_{p, q}(-x)=1$. For $m, n \in \mathbb{N}$, the $(p, q)$-Beta function of second kind and $(p, q)$-Gamma function are defined by

$$
B_{p, q}(m, n)=\int_{0}^{\infty} \frac{x^{m-1}}{(1 \oplus p x)_{p, q}^{m+n}} \mathrm{~d}_{p, q} x
$$

and

$$
\Gamma_{p, q}(n)=\int_{0}^{\infty} p^{\frac{(n-1)(n-2)}{2}} x^{n-1} E_{p, q}(-q x) \mathrm{d}_{p, q} x, \Gamma_{p, q}(n+1)=[n]_{p, q} !
$$


respectively. Then the two functions defined above satisfy the following relation

$$
B_{p, q}(m, n)=\frac{q \Gamma_{p, q}(m) \Gamma_{p, q}(n)}{\left(p^{m+1} q^{m-1}\right)^{\frac{m}{2}} \Gamma_{p, q}(m+n)} .
$$

In particular, if $p=q=1$, then

$$
B(m, n)=\frac{\Gamma(m) \Gamma(n)}{\Gamma(m+n)} .
$$

It may be observed that, in the $(p, q)$-setting, the order is important, which serves the reason why the $(p, q)$-variant of the Beta function does not satisfy the commutativity property, i.e., $B_{p, q}(m, n) \neq B_{p, q}(n, m)$. For $p=1$, all the notions of the $(p, q)$-calculus are reduced to those of the $q$-calculus.

Now, we construct the modified $(p, q)$-Gamma operators preserving linear functions as

Definition 1.1. For $n \in \mathbb{N}, x \in(0, \infty)$ and $0<q<p \leq 1$, the modified $(p, q)$-Gamma operators can be defined as

$$
\widehat{G}_{n}^{p, q}(f ; x)=\frac{\left(\frac{[n+2]_{p, q} x}{[n+1]_{p, q}}\right)^{n+3} q^{n^{2}+4 n+3} p^{n^{2}+3 n+2}}{B_{p, q}(n+1, n+3)} \int_{0}^{\infty} \frac{t^{n}}{\left(\frac{(p q)^{n+1}[n+2]_{p, q} x}{[n+1]_{p, q}} \oplus t\right)_{p, q}^{2 n+4}} f(t) \mathrm{d}_{p, q} t .
$$

Note that, for $q \rightarrow 1^{-}$and $p \rightarrow 1^{-}, \widehat{G}_{n}^{1^{-}, 1^{-}}(f ; x)$ is reduced to the Gamma operators defined in (1.1); for $p \rightarrow 1^{-}, \widehat{G}_{n}^{1^{-}, q}(f ; x)$ is reduced to the modified $q$-Gamma operators defined in [4]. For the results on Gamma type operators or $(p, q)$-operators, we can refer to [15, 16, 17, 18, 19, 20].

The paper is organized as follows. In Section 2, we present the moments of the operators. In Section 3 and Section 4, we study the local approximation and the rate of convergence for these operators. In Section 5 and Section 6, we establish the weighted approximation and the Voronovskaya type theorem.

\section{Moments Estimates}

In order to obtain the approximation properties of the operators $\widehat{G}_{n}^{p, q}(f ; x)$, we need the following lemmas.

Lemma 2.1. For any $n \in \mathbb{N}, x \in(0, \infty)$ and $0<q<p \leq 1$, the following equalities hold:

1. $\widehat{G}_{n}^{p, q}(1 ; x)=1$;

2. $\widehat{G}_{n}^{p, q}(t ; x)=x$;

3. $\widehat{G}_{n}^{p, q}\left(t^{2} ; x\right)=\frac{[n+2]_{p, q}^{2}}{p q[n+1]_{p, q}^{2}} x^{2}$;

4. $\widehat{G}_{n}^{p, q}\left(t^{3} ; x\right)=\frac{[n+3]_{p, q}[n+2]_{p, q}^{3}}{(p q)^{3}[n]_{p, q}[n+1]_{p, q}^{3}} x^{3}$;

5. $\widehat{G}_{n}^{p, q}\left(t^{4} ; x\right)=\frac{[n+3]_{p, q}[n+4]_{p, q}[n+2]_{p, q}^{4}}{(p q)^{6}[n]_{p, q}[n-1]_{p, q}[n+1]_{p, q}^{4}} x^{4}$, for $n>1$. 
Proof. Using the properties of the $(p, q)$-Beta function of second kind and the $(p, q)$-Gamma function with $k \leq n-3$, we can obtain

$$
\begin{aligned}
\widehat{G}_{n}^{p, q}\left(t^{k} ; x\right)= & \frac{\left(\frac{[n+2]_{p, q} x}{[n+1]_{p, q}}\right)^{n+3} q^{n^{2}+4 n+3} p^{n^{2}+3 n+2}}{B_{p, q}(n+1, n+3)} \int_{0}^{\infty} \frac{t^{n+k}}{\left(\frac{(p q)^{n+1}[n+2]_{p, q} x}{[n+1]_{p, q}} \oplus\right)_{p, q}^{2 n+4}} \mathrm{~d}_{p, q} t \\
= & \left(\frac{[n+2]_{p, q} x}{[n+1]_{p, q}}\right)^{-n-1} \frac{q^{n^{2}+4 n+3} p^{n^{2}+3 n+2}}{B_{p, q}(n+1, n+3)} \\
& \times \int_{0}^{\infty} \frac{1}{(p q)^{2 n^{2}+6 n+4}} \frac{t^{n+k}}{\left(1 \oplus p\left(\frac{[n+1]_{p, q}}{p^{n+2} q^{n+1}[n+2]_{p, q} x}\right)\right)_{p, q}^{2 n+4}} \mathrm{~d}_{p, q} t \\
= & \left(\frac{[n+2]_{p, q}}{[n+1]_{p, q}}\right)^{k} \frac{p^{2 n^{2}+(k+6) n+2 k+4} q^{n^{2}+(k+6) n+k+4} B_{p, q}(n+k+1, n-k+3)}{(p q)^{2 n^{2}+6 n+4}} \\
= & \frac{(p q)^{\frac{k-k^{2}}{2}}[n+k]_{p, q} ![n-k+2]_{p, q} !}{[n]_{p, q} ![n+2]_{p, q} !}\left(\frac{[n+2]_{p, q}}{[n+1]_{p, q}}\right)^{k} x^{k} .
\end{aligned}
$$

Remark 2.2. For $x \in(0, \infty), 0<q<p \leq 1$, we conclude from Lemma 2.1 that

$$
\begin{array}{r}
\widehat{G}_{n}^{p, q}(t-x ; x)=0, \\
\alpha_{n}^{p, q}(x):=\widehat{G}_{n}^{p, q}\left((t-x)^{2} ; x\right)=\left(\frac{[n+2]_{p, q}^{2}}{p q[n+1]_{p, q}^{2}}-1\right) x^{2} .
\end{array}
$$

Lemma 2.3. If $\left(p_{n}\right)$ and $\left(q_{n}\right)$ satisfy $0<q_{n}<p_{n} \leq 1$, where $p_{n} \rightarrow 1, q_{n} \rightarrow 1$ and $p_{n}^{n} \rightarrow a$, $q_{n}^{n} \rightarrow b,[n]_{p_{n}, q_{n}} \rightarrow \infty$ as $n \rightarrow \infty$, then

1. $\lim _{n \rightarrow \infty}[n+1]_{p_{n}, q_{n}} \widehat{G}_{n}^{p_{n}, q_{n}}\left((t-x)^{2} ; x\right)=(a+b) x^{2}$;

2. $\lim _{n \rightarrow \infty}[n+1]_{p_{n}, q_{n}} \widehat{G}_{n}^{p_{n}, q_{n}}\left((t-x)^{4} ; x\right)=0$.

Proof. 1. By $[n+2]_{p_{n}, q_{n}}=p_{n}^{n+1}+q_{n}[n+1]_{p_{n}, q_{n}}$, we have

$$
\frac{[n+2]_{p_{n}, q_{n}}}{q_{n}[n+1]_{p_{n}, q_{n}}}=1+\frac{p_{n}^{n+1}}{q_{n}[n+1]_{p_{n}, q_{n}}} .
$$


Hence

$$
\begin{aligned}
& \lim _{n \rightarrow \infty}[n+2]_{p_{n}, q_{n}} \widehat{G}_{n}^{p_{n}, q_{n}}\left((t-x)^{2} ; x\right) \\
& =\lim _{n \rightarrow \infty}[n+2]_{p_{n}, q_{n}}\left(\frac{[n+2]_{p_{n}, q_{n}}^{2}}{p_{n} q_{n}[n+1]_{p_{n}, q_{n}}^{2}}-1\right) x^{2} \\
& =\lim _{n \rightarrow \infty}[n+2]_{p_{n}, q_{n}}\left(\frac{q_{n}}{p_{n}}\left(1+\frac{2 p_{n}^{n+1}}{q_{n}[n+1]_{p_{n}, q_{n}}}+\frac{p_{n}^{2 n+2}}{q_{n}^{2}[n+1]_{p_{n}, q_{n}}^{2}}\right)-1\right) x^{2} \\
& =\lim _{n \rightarrow \infty}[n+2]_{p_{n}, q_{n}}\left(\frac{q_{n}-p_{n}}{p_{n}}+\frac{2 p_{n}^{n}}{[n+1]_{p_{n}, q_{n}}}\right) x^{2} \\
& =\lim _{n \rightarrow \infty}[n+2]_{p_{n}, q_{n}}\left(q_{n}-p_{n}\right) x^{2}+2 a x^{2}=(b-a) x^{2}+2 a x^{2}=(a+b) x^{2} .
\end{aligned}
$$

2. In view of

$$
\frac{[n+2]_{p_{n}, q_{n}}}{q_{n}[n+1]_{p_{n}, q_{n}}}=1+\frac{p_{n}^{n+1}}{q_{n}[n+1]_{p_{n}, q_{n}}}
$$

and

$$
[n+m]_{p_{n}, q_{n}}=q_{n}^{m}[n]_{p_{n}, q_{n}}+p_{n}^{n}[m]_{p_{n}, q_{n}},
$$

we easily obtain

$$
\left(\frac{[n+2]_{p_{n}, q_{n}}}{q_{n}[n+1]_{p_{n}, q_{n}}}\right)^{2} \sim 1+\frac{2 p_{n}^{n+1}}{q_{n}[n+1]_{p_{n}, q_{n}}},\left(\frac{[n+2]_{p_{n}, q_{n}}}{q_{n}[n+1]_{p_{n}, q_{n}}}\right)^{3} \sim 1+\frac{3 p_{n}^{n+1}}{q_{n}[n+1]_{p_{n}, q_{n}}}
$$

and

$$
\begin{aligned}
\left(\frac{[n+2]_{p_{n}, q_{n}}}{q_{n}[n+1]_{p_{n}, q_{n}}}\right)^{4} \sim 1+\frac{4 p_{n}^{n+1}}{q_{n}[n+1]_{p_{n}, q_{n}}}, \frac{[n+4]_{p_{n}, q_{n}}}{[n-1]_{p_{n}, q_{n}}} & =q_{n}^{5}+\frac{p_{n}^{n-1}[5]_{p_{n}, q_{n}}}{[n-1]_{p_{n}, q_{n}}}, \frac{[n+3]_{p_{n}, q_{n}}}{[n]_{p_{n}, q_{n}}} \\
& =q_{n}^{3}+\frac{p_{n}^{n}[3]_{p_{n}, q_{n}}}{[n]_{p_{n}, q_{n}}} .
\end{aligned}
$$

Using Lemma 2.1., we can obtain

$$
\begin{aligned}
\widehat{G}_{n}^{p_{n}, q_{n}}\left(t^{2} ; x\right) & \sim\left(\frac{q_{n}}{p_{n}}+\frac{2 p_{n}^{n}}{[n+1]_{p_{n}, q_{n}}}\right) x^{2} \\
\widehat{G}_{n}^{p_{n}, q_{n}}\left(t^{3} ; x\right) & \sim\left(\frac{q_{n}^{3}}{p_{n}^{3}}+\frac{[3]_{p_{n}, q_{n}} p_{n}^{n-3}}{[n]_{p_{n}, q_{n}}}+\frac{3 q_{n}^{2} p_{n}^{n-2}}{[n+1]_{p_{n}, q_{n}}}\right) x^{3} ; \\
\widehat{G}_{n}^{p_{n}, q_{n}}\left(t^{4} ; x\right) & \sim\left(\frac{q_{n}^{6}}{p_{n}^{6}}+\frac{[5]_{p_{n}, q_{n}} p_{n}^{n-7} q_{n}}{[n-1]_{p_{n}, q_{n}}}+\frac{[3]_{p_{n}, q_{n}} p_{n}^{n-6} q_{n}^{3}}{[n]_{p_{n}, q_{n}}}+\frac{4 p_{n}^{n-5} q_{n}^{5}}{[n+1]_{p_{n}, q_{n}}}\right) x^{4} .
\end{aligned}
$$

From the fact that

$$
\begin{aligned}
\widehat{G}_{n}^{p_{n}, q_{n}}\left((t-x)^{4} ; x\right)= & \widehat{G}_{n}^{p_{n}, q_{n}}\left(t^{4} ; x\right)-4 \widehat{G}_{n}^{p_{n}, q_{n}}\left(t^{3} ; x\right) x+6 \widehat{G}_{n}^{p_{n}, q_{n}}\left(t^{2} ; x\right) x^{2} \\
& -4 \widehat{G}_{n}^{p_{n}, q_{n}}(t ; x) x^{3}+\widehat{G}_{n}^{p_{n}, q_{n}}(1 ; x) x^{4}
\end{aligned}
$$


we have

$$
\begin{aligned}
\widehat{G}_{n}^{p_{n}, q_{n}}\left((t-x)^{4} ; x\right) \sim & \left(\frac{q_{n}^{6}}{p_{n}^{6}}-\frac{4 q_{n}^{3}}{p_{n}^{3}}+\frac{6 q_{n}}{p_{n}}-3+\frac{[5]_{p_{n}, q_{n}} p_{n}^{n-7} q_{n}}{[n-1]_{p_{n}, q_{n}}}\right. \\
& \left.+\frac{[3]_{p_{n}, q_{n}} p_{n}^{n-6}\left(q_{n}^{3}-4 p_{n}^{3}\right)}{[n]_{p_{n}, q_{n}}}+\frac{p_{n}^{n-5}\left(4 q_{n}^{5}-12 p_{n}^{3} q_{n}^{2}+12 p_{n}^{5}\right)}{[n+1]_{p_{n}, q_{n}}}\right) x^{4}
\end{aligned}
$$

By combining

$$
\begin{aligned}
& \lim _{n \rightarrow \infty}[n+1]_{p_{n}, q_{n}}\left(\frac{q_{n}^{6}}{p_{n}^{6}}-\frac{4 q_{n}^{3}}{p_{n}^{3}}+\frac{6 q_{n}}{p_{n}}-3\right) \\
& =-\lim _{n \rightarrow \infty}[n+1]_{p_{n}, q_{n}}\left(3 p_{n}^{6}-6 p_{n}^{5} q_{n}+4 p_{n}^{3} q_{n}^{3}-q_{n}^{6}\right) \\
& =-\lim _{n \rightarrow \infty}[n+1]_{p_{n}, q_{n}}\left(p_{n}-q_{n}\right)\left(3[6]_{p_{n}, q_{n}}-6[5]_{p_{n}, q_{n}} q_{n}+4[3]_{p_{n}, q_{n}} q_{n}^{3}\right) \\
& =-(a-b)(3 \times 6-6 \times 5+4 \times 3)=0
\end{aligned}
$$

with

$$
\begin{aligned}
& \lim _{n \rightarrow \infty}[n+1]_{p_{n}, q_{n}}\left(\frac{[5]_{p_{n}, q_{n}} p_{n}^{n-7} q_{n}}{[n-1]_{p_{n}, q_{n}}}+\frac{[3]_{p_{n}, q_{n}} p_{n}^{n-6}\left(q_{n}^{3}-4 p_{n}^{3}\right)}{[n]_{p_{n}, q_{n}}}+\frac{p_{n}^{n-5}\left(4 q_{n}^{5}-12 p_{n}^{3} q_{n}^{2}+12 p_{n}^{5}\right)}{[n+1]_{p_{n}, q_{n}}}\right) \\
& =5 a+3 \times(1-4) a+(4-12-12) a=0,
\end{aligned}
$$

we complete the proof immediately.

\section{LOCAL APPROXIMATION}

In this section, we present a local approximation theorem of modified $(p, q)$-Gamma operators. Let $C_{B}[0, \infty)$ be the space of all real-valued continuous and bounded functions $f$ defined on the interval $[0, \infty)$, endowed with the norm $\|f\|=\lim _{x \in[0, \infty)}|f(x)|$. Further, the Peetre's $K$ functional is defined by

$$
K(f, \delta)=\inf _{g \in W^{2}}\left\{\|f-g\|+\delta\left\|g^{\prime \prime}\right\|\right\}
$$

where $\delta>0$ and $W^{2}=\left\{g \in C_{B}[0, \infty): g^{\prime}, g^{\prime \prime} \in C_{B}[0, \infty)\right\}$. The usual modulus of continuity and the second-order modulus of smoothness of $f \in C_{B}[0, \infty)$ are defined by

$$
\omega(f, \delta)=\sup _{0<|h| \leq \delta} \sup _{x \in[0, \infty)}|f(x+h)-f(x)| .
$$

and

$$
\omega_{2}(f, \delta)=\sup _{0<|h| \leq \delta} \sup _{x \in[0, \infty)}|f(x+2 h)-2 f(x+h)+f(x)|
$$

From [21], we see that there exists an absolute constant $C>0$ such that

$$
K(f, \delta) \leq C \omega_{2}(f, \sqrt{\delta}) .
$$

Theorem 3.1. Let $f \in C_{B}[0,+\infty), 0<q<p \leq 1$. Then, for every $x \in(0, \infty)$,

$$
\left|\widehat{G}_{n}^{p, q}(f ; x)-f(x)\right| \leq 2 C \omega_{2}\left(f, \sqrt{\alpha_{n}^{p, q}(x)}\right) .
$$


Proof. For all $g \in C_{B}^{2}[0, \infty)$, using the Taylor's expansion for $x, t \in(0, \infty)$, we have

$$
g(t)=g(x)+g^{\prime}(x)(t-x)+\int_{x}^{t}(t-u) g^{\prime \prime}(u) \mathrm{d} u .
$$

Applying the operators $\widehat{G}_{n}^{p, q}$ to both sides of the equality above and using Remark 2.2, we get

$$
\begin{aligned}
\left|\widehat{G}_{n}^{p, q}(g ; x)-g(x)\right| & =\left|\widehat{G}_{n}^{p, q}\left(\int_{x}^{t}(t-u) g^{\prime \prime}(u) \mathrm{d} u ; x\right)\right| \\
& \leq \widehat{G}_{n}^{p, q}\left(\left|\int_{x}^{t}(t-u) g^{\prime \prime}(u) \mathrm{d} u\right| ; x\right) \\
& \leq \widehat{G}_{n}^{p, q}\left(\left\|g^{\prime \prime}\right\|(t-x)^{2} ; x\right) \\
& \leq \alpha_{n}^{p, q}(x)\left\|g^{\prime \prime}\right\| .
\end{aligned}
$$

Using $\left|\widehat{G}_{n}^{p, q}(f ; x)\right| \leq\|f\|$, we have

$$
\begin{aligned}
\left|\widehat{G}_{n}^{p, q}(f ; x)-f(x)\right| & \leq\left|\widehat{G}_{n}^{p, q}(f-g ; x)-(f-g)(x)\right|+\left|\widehat{G}_{n}^{p, q}(g ; x)-g(x)\right| \\
& \leq 2\|f-g\|+\alpha_{n}^{p, q}(x)\left\|g^{\prime \prime}\right\|
\end{aligned}
$$

By taking infimum on both sides of the inequality above over all $g \in C_{B}^{2}[0, \infty)$

$$
\left|\widehat{G}_{n}^{p, q}(f ; x)-f(x)\right| \leq 2 K\left(f ; \alpha_{n}^{p, q}(x)\right),
$$

and using (3.1), we conclude the desired conclusion immediately.

\section{RAte of CONVERGEnCE}

Let $B_{2}[0, \infty)$ be the set of all functions $f$ defined on $[0, \infty)$ satisfying the condition $|f(x)| \leq$ $M_{f}\left(1+x^{2}\right)$ with some absolute constant $M_{f}>0$ which depends on $f$ only. $C_{2}[0, \infty)$ denotes the subspace of all continuous functions $f \in B_{2}[0, \infty)$ with the norm

$$
\|f\|_{2}=\sup _{x \in[0, \infty)} \frac{|f(x)|}{1+x^{2}} \text {. }
$$

By $C_{2}^{0}[0, \infty)$, we denote the subspace of all functions $f \in C_{2}[0, \infty)$ for which $\lim _{n \rightarrow \infty} \frac{|f(x)|}{1+x^{2}}$ is finite. Meantime, we denote the modulus of continuity of $f$ on the closed interval $[0, a], a>0$ by

$$
\omega_{a}(f, \delta)=\sup _{|t-x| \leq \delta} \sup _{x, t \in[0, a]}|f(t)-f(x)| .
$$

Obviously, for function $f \in C_{2}[0, \infty)$, the modulus of continuity $\omega_{a}(f, \delta) \rightarrow 0$ as $\delta \rightarrow 0$. Then, we establish the following theorem on the rate of convergence of operators $\widehat{G}_{n}^{p, q}(f ; x)$.

Theorem 4.1. Let $f \in C_{2}^{0}[0, \infty)$ and $0<q<p \leq 1$. Let $\omega_{a+1}(f, \delta)$ be the modulus of continuity on $[0, a+1] \subset[0, \infty)$, where $a>0$. Then,

$$
\left\|\widehat{G}_{n}^{p, q}(f)-f\right\|_{C(0, a]} \leq 4 M_{f}\left(1+a^{2}\right) \alpha_{n}^{p, q}(a)+2 \omega_{a+1}\left(f, \sqrt{\alpha_{n}^{p, q}(a)}\right) .
$$


Proof. For all $x \in(0, a]$ and $t>a+1$, we easily have $(t-x)^{2} \geq(t-a)^{2} \geq 1$. Therefore,

$$
\begin{aligned}
|f(t)-f(x)| & \leq|f(t)|+|f(x)| \\
& \leq M_{f}\left(2+x^{2}+t^{2}\right) \\
& =M_{f}\left(2+x^{2}+(x-t-x)^{2}\right) \\
& \leq M_{f}\left(2+3 x^{2}+2(x-t)^{2}\right) \\
& \leq M_{f}\left(4+3 x^{2}\right)(t-x)^{2} \\
& \leq 4 M_{f}\left(1+a^{2}\right)(t-x)^{2} .
\end{aligned}
$$

For all $x \in(0, a], t \in(0, a+1]$ and $\delta>0$, we have

$$
|f(t)-f(x)| \leq \omega_{a+1}(f,|t-x|) \leq\left(1+\frac{|t-x|}{\delta}\right) \omega_{a+1}(f, \delta) .
$$

From (4.1) and (4.2), we get

$$
|f(t)-f(x)| \leq 4 M_{f}\left(1+a^{2}\right)(t-x)^{2}+\left(1+\frac{|t-x|}{\delta}\right) \omega_{a+1}(f, \delta) .
$$

By Schwarz's inequality and Remark 2.2, for all $x \in(0, a]$, we have

$$
\begin{aligned}
& \left|\widehat{G}_{n}^{p, q}(f ; x)-f(x)\right| \\
& \leq \widehat{G}_{n}^{p, q}(|f(t)-f(x)| ; x) \\
& \leq 4 M_{f}\left(1+a^{2}\right) \widehat{G}_{n}^{p, q}\left((t-x)^{2} ; x\right)+\widehat{G}_{n}^{p, q}\left(\left(1+\frac{|t-x|}{\delta}\right) ; x\right) \omega_{a+1}(f, \delta) \\
& \leq 4 M_{f}\left(1+a^{2}\right) \widehat{G}_{n}^{p, q}\left((t-x)^{2} ; x\right)+\omega_{a+1}(f, \delta)\left(1+\frac{1}{\delta} \sqrt{\widehat{G}_{n}^{p, q}\left((t-x)^{2} ; x\right)}\right) \\
& \leq 4 M_{f}\left(1+a^{2}\right) \alpha_{n}^{p, q}(x)+\omega_{a+1}(f, \delta)\left(1+\frac{1}{\delta} \sqrt{\alpha_{n}^{p, q}(x)}\right) \\
& \leq 4 M_{f}\left(1+a^{2}\right) \alpha_{n}^{p, q}(a)+\omega_{a+1}(f, \delta)\left(1+\frac{1}{\delta} \sqrt{\alpha_{n}^{p, q}(a)}\right) .
\end{aligned}
$$

Choosing $\delta=\sqrt{\alpha_{n}^{p, q}(a)}$, we have the desired results easily.

Next, we establish two point-wise estimates on the rate of convergence of modified $(p, q)$ Gamma operators. First, we recall that a function $f \in C[0, \infty)$ is said to satisfy Lipschitz condition $\operatorname{Lip}_{\gamma}$ on $D, \gamma \in(0,1], D \subset[0, \infty)$ if

$$
|f(t)-f(x)| \leq M_{f, \gamma}|t-x|^{\alpha}, t \in[0, \infty) \text { and } x \in D,
$$

where $M_{f, \gamma}$ is an absolute positive constant depending only on $f$ and $\gamma$.

Theorem 4.2. Let $f \in \operatorname{Lip}_{\gamma}$ on $D, \gamma \in(0,1]$, and $D \subset[0, \infty)$. Then, for all $x \in(0, \infty)$,

$$
\left|\widehat{G}_{n}^{p, q}(f ; x)-f(x)\right| \leq M_{f, \gamma}\left(\left(\alpha_{n}^{p, q}(x)\right)^{\frac{\gamma}{2}}+2 d^{\gamma}(x ; D)\right)
$$

where $d(x ; D)$ represents the distance between $x$ and $D$. 
Proof. From the properties of the infimum, we see that there exists at least a point $x_{0}$ in the closure of $D$, that is, $x_{0} \in \bar{D}$ such that

$$
\mathrm{d}(x ; D)=\left|x_{0}-x\right|
$$

Using

$$
|f(t)-f(x)| \leq\left|f(t)-f\left(x_{0}\right)\right|+\left|f\left(x_{0}\right)-f(x)\right|, x, t \in(0, \infty),
$$

we have

$$
\begin{aligned}
\left|\widehat{G}_{n}^{p, q}(f ; x)-f(x)\right| & \leq M_{f, \gamma}\left(\widehat{G}_{n}^{p, q}\left(\left|t-x_{0}\right|^{\gamma} ; x\right)+\widehat{G}_{n}^{p, q}\left(\left|x_{0}-x\right|^{\gamma} ; x\right)\right) \\
& \leq M_{f, \gamma}\left(\widehat{G}_{n}^{p, q}\left(|t-x|^{\gamma} ; x\right)+2\left|x_{0}-x\right|^{\gamma}\right)
\end{aligned}
$$

Applying the well-known Hölder inequality with $k_{1}=\frac{2}{\gamma}$ and $k_{2}=\frac{2}{2-\gamma}$, we have

$$
\begin{aligned}
\left|\widehat{G}_{n}^{p, q}(f ; x)-f(x)\right| & \leq M_{f, \gamma}\left\{\left(\widehat{G}_{n}^{p, q}\left(|t-x|^{\gamma k_{1}} ; x\right)\right)^{\frac{1}{k_{1}}}\left(\widehat{G}_{n}^{p, q}\left(1^{k_{2}} ; x\right)\right)^{\frac{1}{k_{2}}}+2\left|t_{0}-x\right|^{\gamma}\right\} \\
& \leq M_{f, \gamma}\left\{\left(\widehat{G}_{n}^{p, q}\left((t-x)^{2} ; x\right)\right)^{\frac{\gamma}{2}}+2\left|t_{0}-x\right|^{\gamma}\right\} \\
& \leq M_{f, \gamma}\left\{\left(\alpha_{n}^{p, q}(x)\right)^{\frac{\gamma}{2}}+2(\mathrm{~d}(x ; D))^{\gamma}\right\} .
\end{aligned}
$$

This completes the proof.

Second, we give the local direct estimate of modified $(p, q)$-Gamma operators by using the Lipschitz-type maximal functions of order $\gamma$, introduced by Lenze [22] as

$$
\widetilde{\omega}_{\gamma}(f ; x)=\sup _{t \in[0, \infty), t \neq x} \frac{|f(t)-f(x)|}{|t-x|^{\gamma}}, x \in[0, \infty) \text { and } \gamma \in(0,1] \text {. }
$$

Theorem 4.3. Let $0<q<p \leq 1$. Then, for all $f \in C_{B}[0, \infty)$,

$$
\left|\widehat{G}_{n}^{p, q}(f ; x)-f(x)\right| \leq \widetilde{\omega}_{\gamma}(f ; x)\left(\alpha_{n}^{p, q}(x)\right)^{\frac{\gamma}{2}} \text {. }
$$

Proof. Using the monotonicity of the operators $\widehat{G}_{n}^{p, q}$ and the Hölder inequality, we can obtain

$$
\begin{aligned}
\left|\widehat{G}_{n}^{p, q}(f ; x)-f(x)\right| & \leq \widehat{G}_{n}^{p, q}(|f(t)-f(x)|) \leq \widetilde{\omega}_{\gamma}(f ; x) \widehat{G}_{n}^{p, q}\left(|t-x|^{\gamma} ; x\right) \\
& \leq \widetilde{\omega}_{\gamma}(f ; x)\left(\widehat{G}_{n}^{p, q}\left((t-x)^{2} ; x\right)\right)^{\frac{\gamma}{2}}=\widetilde{\omega}_{\gamma}(f ; x)\left(\alpha_{n}^{p, q}(x)\right)^{\frac{\gamma}{2}} .
\end{aligned}
$$

This completes the proof.

\section{Weighted APPROXimATiON}

In this section, we discuss the weighted approximation theorems of the operators (1.1). In [23], Yuksel and Ispir defined the weighted modulus of continuity

$$
\Omega(f ; \delta)=\sup _{0<h \leq \delta, x \geq 0} \frac{|f(x+h)-f(x)|}{1+(x+h)^{2}}
$$

where $f \in C_{2}^{0}[0, \infty)$ and proved the properties of monotone increasing about $\Omega(f ; \delta)$ as $\delta>0$ and the inequality $\Omega(f ; \lambda \delta) \leq(1+\lambda) \Omega(f ; \delta)$, where $\lambda>0$ and $f \in C_{2}^{0}[0, \infty)$. 
Theorem 5.1. Let $f \in C_{2}^{0}[0, \infty)$, and let $\left(p_{n}\right)$ and $\left(q_{n}\right)$ satisfy $0<q_{n}<p_{n} \leq 1$ such that $p_{n}^{n} \rightarrow 1$, $q_{n}^{n} \rightarrow 1$ and $[n]_{p_{n}, q_{n}} \rightarrow \infty$ as $n \rightarrow \infty$. Then, there exists a positive integer $N \in \mathbb{N}_{+}$such that, for all $n>N$ and $v>0$, the inequality

$$
\sup _{x \in(0, \infty)} \frac{\left|\widehat{G}_{n}^{p_{n}, q_{n}}(f ; x)-f(x)\right|}{\left(1+x^{2}\right)^{\frac{3}{2}+v}} \leq 4 \sqrt{2} \Omega\left(f ; \frac{1}{\sqrt{[n+1]_{p_{n}, q_{n}}}}\right)
$$

holds.

Proof. For $x, t \in(0, \infty)$ and $\delta>0$, by the definition and properties of $\Omega(f ; \delta)$, we get

$$
\begin{aligned}
|f(t)-f(x)| & \leq(1+(x+|x-t|))^{2} \Omega(f ;|t-x|) \\
& \leq 2\left(1+x^{2}\right)\left(1+(t-x)^{2}\right)\left(1+\frac{|t-x|}{\delta}\right) \Omega(f ; \delta) .
\end{aligned}
$$

Using the fact that $p_{n}^{n} \rightarrow 1, q_{n}^{n} \rightarrow 1$ and $[n]_{p_{n}, q_{n}} \rightarrow \infty$ as $n \rightarrow \infty$ and Lemma 2.3, we find that there exists a positive integer $N \in \mathbb{N}_{+}$such that, for all $n>N$,

$$
\begin{gathered}
\widehat{G}_{n}^{p_{n}, q_{n}}\left((t-x)^{2} ; x\right) \leq \frac{2\left(1+x^{2}\right)}{[n+1]_{p_{n}, q_{n}}}, \\
\widehat{G}_{n}^{p_{n}, q_{n}}\left((t-x)^{4} ; x\right) \leq 1,
\end{gathered}
$$

Since $\widehat{G}_{n}^{p_{n}, q_{n}}$ is linear and positive, we have

$$
\begin{array}{r}
\left|\widehat{G}_{n}^{p_{n}, q_{n}}(f ; x)-f(x)\right| \leq 2\left(1+x^{2}\right) \Omega(f ; \delta)\left\{1+\widehat{G}_{n}^{p_{n}, q_{n}}\left((t-x)^{2} ; x\right)+\right. \\
\left.\widehat{G}_{n}^{p_{n}, q_{n}}\left(\left(1+(t-x)^{2}\right) \frac{|t-x|}{\delta} ; x\right)\right\} .
\end{array}
$$

To estimate the second term of (5.4), we find from the Cauchy-Schwartz inequality and $(x+$ $y)^{2} \leq 2\left(x^{2}+y^{2}\right)$ that

$$
\widehat{G}_{n}^{p_{n}, q_{n}}\left(\left(1+(t-x)^{2}\right) \frac{|t-x|}{\delta} ; x\right) \leq \sqrt{2}\left(\widehat{G}_{n}^{p_{n}, q_{n}}\left(1+(t-x)^{4} ; x\right)\right)^{\frac{1}{2}}\left(\widehat{G}_{n}^{p_{n}, q_{n}}\left(\frac{(t-x)^{2}}{\delta^{2}} ; x\right)\right)^{\frac{1}{2}} .
$$

Combining (5.2) and (5.3), we arrive at

$$
\widehat{G}_{n}^{p_{n}, q_{n}}\left(\left(1+(t-x)^{2}\right) \frac{|t-x|}{\delta} ; x\right) \leq \frac{2 \sqrt{2}\left(1+x^{2}\right)^{\frac{1}{2}}}{\delta[n+1]_{p_{n}, q_{n}}} .
$$

Taking $\delta=\frac{1}{\sqrt{[n+1]_{p_{n}, q_{n}}}}$, we can obtain

$$
\left|\widehat{G}_{n}^{p_{n}, q_{n}}(f ; x)-f(x)\right| \leq 4 \sqrt{2}\left(1+x^{2}\right)^{\frac{3}{2}} \Omega\left(f ; \frac{1}{\sqrt{[n+1]_{p_{n}, q_{n}}}}\right) .
$$

The proof is completed.

Theorem 5.2. Let the sequences $\left(p_{n}\right),\left(q_{n}\right)$ satisfy $0<q_{n}<p_{n} \leq 1$ such that $p_{n} \rightarrow 1, q_{n} \rightarrow 1$ and $p_{n}^{n} \rightarrow a, q_{n}^{n} \rightarrow b,[n]_{p_{n}, q_{n}} \rightarrow \infty$ as $n \rightarrow \infty$. Then, for $f \in C_{2}^{0}[0, \infty)$, we have

$$
\lim _{n \rightarrow \infty}\left\|\widehat{G}_{n}^{p_{n}, q_{n}}(f)-f\right\|_{2}=0 \text {. }
$$


Proof. By the Korovkin theorem in [24], we see that it is sufficient to verify the following three conditions

$$
\lim _{n \rightarrow \infty}\left\|\widehat{G}_{n}^{p_{n}, q_{n}}\left(t^{k} ; x\right)-x^{k}\right\|_{2}=0, k=0,1,2 .
$$

Since $\widehat{G}_{n}^{p_{n}, q_{n}}(1 ; x)=1$ and $\widehat{G}_{n}^{p_{n}, q_{n}}(t ; x)=x$, then (5.6) holds true for $k=0,1$. By Lemma 2.3, we get

$$
\begin{aligned}
\left\|\widehat{G}_{n}^{p_{n}, q_{n}}\left(t^{2} ; x\right)-x^{2}\right\|_{2} & =\sup _{x \in(0, \infty)} \frac{1}{1+x^{2}}\left|\widehat{G}_{n}^{p_{n}, q_{n}}\left(t^{2} ; x\right)-x^{2}\right| \\
& \leq \sup _{x \in(0, \infty)} \frac{x^{2}}{1+x^{2}} \frac{\left|p_{n}-q_{n}\right|}{p_{n}}+\sup _{x \in(0, \infty)} \frac{x^{2}}{1+x^{2}} \frac{2 p_{n}^{n}}{[n+1]_{p_{n}, q_{n}}} \\
& \leq \frac{\left|p_{n}-q_{n}\right|}{p_{n}}+\frac{2 p_{n}^{n}}{[n+1]_{p_{n}, q_{n}}} \rightarrow 0, n \rightarrow \infty .
\end{aligned}
$$

Thus the proof is completed.

Theorem 5.3. Let the sequences $\left(p_{n}\right),\left(q_{n}\right)$ satisfy $0<q_{n}<p_{n} \leq 1$ such that $p_{n} \rightarrow 1, q_{n} \rightarrow 1$, $[n]_{p_{n}, q_{n}} \rightarrow \infty$ as $n \rightarrow \infty$. For every $f \in C_{2}[0, \infty)$ and $\kappa>0$, we have

$$
\lim _{n \rightarrow \infty} \sup _{x \in(0, \infty)} \frac{\left|\widehat{G}_{n}^{p_{n}, q_{n}}(f ; x)-f(x)\right|}{\left(1+x^{2}\right)^{1+\kappa}}=0 .
$$

Proof. Let $x_{0} \in[0, \infty)$ be arbitrarily fixed. Then

$$
\begin{aligned}
\sup _{x \in(0, \infty)} \frac{\left|\widehat{G}_{n}^{p_{n}, q_{n}}(f ; x)-f(x)\right|}{\left(1+x^{2}\right)^{1+\kappa}} & \leq \sup _{x \in\left(0, x_{0}\right]} \frac{\left|\widehat{G}_{n}^{p_{n}, q_{n}}(f ; x)-f(x)\right|}{\left(1+x^{2}\right)^{1+\kappa}} \\
& +\sup _{x \in\left(x_{0}, \infty\right)} \frac{\left|\widehat{G}_{n}^{p_{n}, q_{n}}(f ; x)-f(x)\right|}{\left(1+x^{2}\right)^{1+\kappa}} \\
& \leq\left\|\widehat{G}_{n}^{p_{n}, q_{n}}(f ; x)-f(x)\right\|_{C\left[0, x_{0}\right]} \\
& +M_{f} \sup _{x \in\left(x_{0}, \infty\right)} \frac{\left|\widehat{G}_{n}^{p_{n}, q_{n}}\left(\left(1+t^{2}\right) ; x\right)\right|}{\left(1+x^{2}\right)^{1+\kappa}} \\
& +\sup _{x \in\left(x_{0}, \infty\right)} \frac{|f(x)|}{\left(1+x^{2}\right)^{1+\kappa}}
\end{aligned}
$$

Since $|f(x)| \leq M_{f}\left(1+x^{2}\right)$, we have

$$
\sup _{x \in\left(x_{0}, \infty\right)} \frac{|f(x)|}{\left(1+x^{2}\right)^{1+\kappa}} \leq \frac{M_{f}}{\left(1+x_{0}^{2}\right)^{\kappa}} .
$$

Let $\varepsilon>0$ be arbitrary. We can choose $x_{0}$ large enough such that

$$
\frac{M_{f}}{\left(1+x_{0}^{2}\right)^{\kappa}}<\varepsilon
$$

In view of Lemma 2.1, we obtain, $x \in\left(x_{0}, \infty\right)$,

$$
M_{f} \lim _{n \rightarrow \infty} \frac{\left|\widehat{G}_{n}^{p_{n}, q_{n}}\left(\left(1+t^{2}\right) ; x\right)\right|}{\left(1+x^{2}\right)^{1+\kappa}}=M_{f} \frac{\left(1+x^{2}\right)}{\left(1+x^{2}\right)^{1+\kappa}}=\frac{M_{f}}{\left(1+x^{2}\right)^{\kappa}} \leq \frac{M_{f}}{\left(1+x_{0}^{2}\right)^{\kappa}}<\varepsilon .
$$


Using Theorem 4.1, we can see that the first term of inequality (5.7) implies that

$$
\left\|\widehat{G}_{n}^{p_{n}, q_{n}}(f)-f\right\|_{C\left(0, x_{0}\right]}<\varepsilon, \text { as } n \rightarrow \infty .
$$

Combining (5.7), (5.8) and (5.9), we obtain the desired result immediately.

\section{VORONOVSKAYA TYPE THEOREM}

In this section, we give a Voronovskaya type asymptotic formula for $\widehat{G}_{n}^{p_{n}, q_{n}}(f ; x)$ by means of the second and fourth central moments.

Theorem 6.1. Let $f$ be bounded and integrable on the interval $x \in(0, \infty)$. Assume that second derivative of $f$ exists at a fixed point $x \in(0, \infty)$, and the sequences $\left(p_{n}\right)$ and $\left(q_{n}\right)$ satisfy $0<$ $q_{n}<p_{n} \leq 1$ with $p_{n} \rightarrow 1, q_{n} \rightarrow 1, p_{n}^{n} \rightarrow a, q_{n}^{n} \rightarrow b$, and $[n]_{p_{n}, q_{n}} \rightarrow \infty$ as $n \rightarrow \infty$. Then

$$
\lim _{n \rightarrow \infty}[n+1]_{p_{n}, q_{n}}\left(\widehat{G}_{n}^{p_{n}, q_{n}}(f ; x)-f(x)\right)=\frac{a+b}{2} f^{\prime \prime}(x) x^{2} .
$$

Proof. Use Taylor's expansion

$$
f(t)-f(x)=f^{\prime}(x)(t-x)+\frac{1}{2} f^{\prime \prime}(x)(t-x)^{2}+\Theta(t, x)(t-x)^{2},
$$

where $x>0, t>0, \Theta(t, x)$ is bounded and $\lim _{t \rightarrow x} \Theta(t, x)=0$. Applying the operator $P_{n}^{p_{n}, q_{n}}$ to the equality above, we can obtain

$$
\widehat{G}_{n}^{p_{n}, q_{n}}(f ; x)-f(x)=\frac{1}{2} f^{\prime \prime}(x) \widehat{G}_{n}^{p_{n}, q_{n}}\left((t-x)^{2} ; x\right)+\widehat{G}_{n}^{p_{n}, q_{n}}\left(\Theta(t, x)(t-x)^{2} ; x\right) .
$$

Since $\lim _{t \rightarrow x} \Theta(t, x)=0$, we have that, for all $\varepsilon>0$, there exists $\delta>0$ such that $|t-x|<\delta$ and $|\Theta(t, x)|<\varepsilon$ for all fixed $x \in(0, \infty)$ as $n$ goes sufficiently large. While, if $|t-x| \geq \delta$, then $|\Theta(t, x)| \leq \frac{C}{\delta^{2}}(t-x)^{2}$, where $C>0$ is a constant. Using Lemma 2.3, we have

$$
\begin{aligned}
& {[n+1]_{p_{n}, q_{n}}\left|\widehat{G}_{n}^{p_{n}, q_{n}}\left(\Theta(t, x)(t-x)^{2} ; x\right)\right|} \\
& \leq \varepsilon[n+1]_{p_{n}, q_{n}} \widehat{G}_{n}^{p_{n}, q_{n}}\left((t-x)^{2} ; x\right)+\frac{C}{\delta^{2}}[n]_{p_{n}, q_{n}} \widehat{G}_{n}^{p_{n}, q_{n}}\left((t-x)^{4} ; x\right) \rightarrow 0(n \rightarrow \infty) .
\end{aligned}
$$

The proof is completed.

\section{Funding}

This paper was supported by the National Natural Science Foundation of China (Grant No. 11626031), the Key Natural Science Research Project in Universities of Anhui Province (Grant No. KJ2019A0572 and Grant No. KJ2019A0580), the Philosophy and Social Sciences General Planning Project of Anhui Province of China (Grant No. AHSKYG2017D153) and the Natural Science Foundation of Anhui Province of China (Grant No. 1908085QA29). 


\section{REFERENCES}

[1] H. Karsli, Rate of convergence of a new Gamma type operators for the functions with derivatives of bounded variation, Math. Comput. Modell. 45 (2007), 617-624.

[2] H. Karsli, Özarslan M. Ali, Direct local and global approximation results for the operators of gamma type, Hacet. J. Math. Stat. 39 (2010), 241-253.

[3] Q.B. Cai, X.M. Zeng, On the convergence of a kind of $q$-Gamma operators, J. Inequal. Appl. 2013 (2013), 105.

[4] Q.B. Cai, X.M. Zeng, On the convergence of a kind of a modified $q$-Gamma operators, J. Comput. Anal. Appl. 15 (2013), 826-863.

[5] S.N. Chen, W.T. Cheng, X.M. Zeng, Stancu type generalization of modified Gamma operators based on $q$-integers, Bull. Korean Math. Soc. 54 (2017), 359-373.

[6] C. Zhao, W.T. Cheng, X.M. Zeng, Some approximation properties of a kind of $q$-Gamma Stancu, J. Inequal. Appl. 2014 (2014), 94.

[7] M. Mursaleen, F. Khan, A. Khan, On $(p, q)$-analogue of Bernstein operators. Appl. Math. Comput. 266 (2015), 874-882.

[8] M. Mursaleen, F. Khan, A. Khan, Some approximation results by $(p, q)$-analogue of Bernstein-Stancu operators, Appl. Math. Comput. 264 (2015), 392-402.

[9] Q.B. Cai, G.R. Zhou, On $(p, q)$-analogue of Kantorovich type Bernstein-Stancu-Schurer operators, Appl. Math. Comput. 276 (2016), 12-20.

[10] W.T. Cheng, W.H. Zhang, On $(p, q)$-analogue of Gamma operators, J. Funct. Space 2019 (2019), Article ID 9607517.

[11] A. Aral, V. Gupta, R.P. Agarwal, Application of $q$-Calculus in Operator Theory, Springer, Berlin, 2013.

[12] V. Gupta, R.P. Agarwal, Convergence Estimates in Approximation Theory, Springer, New York, 2014.

[13] V. Gupta, T.M. Rassias, P.N. Agrawal, A.M. Acu, Recent Advances in Constructive Approximation Theory, Springer, New York, 2018.

[14] P.N. Sadjang, On two $(p, q)$-analogues of the Laplace transform, J. Differ. Equ. Appl. 23 (2017), 1562-1583.

[15] T. Acar, $(p, q)$-Generalization of Szász-Mirakyan operators, Math. Meth. Appl. Sci. 39 (2015), 2685-2695.

[16] T. Acar, S. A. Mohiuddine, M. Mursaleen, Approximation by $(p, q)$-Baskakov-Durrmeyer-Stancu operators, Complex Anal. Oper. Theory, 12 (2018), 1453-1468.

[17] T. Acar, M. Mursaleen, S.N. Deveci, Gamma operators reproducing exponential functions, Adv. Difference Equ. 2020 (2020), 423.

[18] S.N. Deveci, T. Acar, O. Alagoz, Approximation by Gamma type operators, Math. Meth. Appl. Sci. 43 (2020), 2772-2782.

[19] H.G.I. Ilarslan, T. Acar, Approximation by bivariate $(p, q)$-Baskakov-Kantorovich operators, Georgian Math. J. 25 (2018), 397-407.

[20] S.A. Mohiuddine, T. Acar, A. Alotaibi, Durrmeyer type $(p, q)$-Baskakov operators preserving linear functions, 24 (2018), 961-973.

[21] R.A. Devore, G.G. Lorentz, Constructive Approximation. Springer. Berlin, 1993.

[22] B. Lenze, On Lipschitz-type maximal functions and their smoothness spaces, Indag. Math. 50 (1988), 53-63.

[23] I. Yuksel, N. Ispir, Weighted approximation by a certain family of summation integral-type operators, Comput. Math. Appl. 52 (2006), 1463-1470.

[24] A.D. Gadzhiev, Theorem of the type of P. P. Korovkin type theorems, Mat. Zametki, 20 (1976), 781-786. 